\title{
Herr J. Büdel und die Gliederung der Würmeiszeit
}

\author{
Von E. C. Kraus, München
}

Als ich 1955 meine Beobachtungen über den jüngeren Würm-interstadialen Verwitterungsboden im Nordalpen-Vorland bei Murnau erstmals veröffentlicht hatte, meldete sich Herr J. BüDEL, Würzburg, zum Wort und griff 1957 diese Beobachtungen und Deutungen an. Er erklärte die Vorkommen als Folgen einer nachwürmischen „rezenten“ Verwitterung. Meine weiteren Ausführungen zu diesem Thema 1961 riefen bei Herrn BüDEL zu meiner Überraschung eine erregte Reaktion hervor. Dabei hielt er es für möglich, auf einigen hektographierten Blättern in deutsch und englisch, die er einer Anzahl von Fachkollegen schickte - mir aber nicht - eine „Richtigstellung" mit persönlichen Vorwürfen gegen mich zu verfassen, so daß der Eindruck entstand, er wolle mich an einer Antwort vor demselben Forum hindern. Diese Art recht zu bekommen überlasse ich dem Urteil meiner Fachkollegen.

Nun, diese Blätter gelangten gleichwohl in meine Hand, so daß ich versuchen kann, unter Hinweis auf die unten zitierten Publikationen demselben Kollegenkreis meine Einstellung bekanntzugeben.

In jenen Blättern lese ich, Herrn BüDELs Arbeiten von 1957 und 1960 hätte ich „auf Grund rein fiktiver Behauptungen in Bausch-und-Bogen abgelehnt", und ich wäre auf seine "darin enthaltenen Beweise und Gedankengänge" mit keinem Worte eingegangen. Das stimmt nun schon gleich nicht. Denn im Gegenteil schrieb ich 1961, S. 44, daß ich mich veranlaßt sähe, „die Gründe zu beleuchten, welche Herrn BüDEL angeregt haben dürften, ein Interstadial zu leugnen und damit gleichzeitig die Klimakurven von P. WoLdstedt (1956, 1958, 1960) abzulehnen“. Herrn BüDELs Gedanken wurden denn auch in meinen Beispielen auf S. 45-47 diskutiert.

Mit Herrn BüDEL gehe ich vielleicht darin einig, daß es wenig Sinn hat, über die verschiedenen, in den Kiesgruben sichtbaren Einzelaufschlüsse angesichts des oft sehr rasch voranschreitenden Grubenabbaues gegeneinander zu geraten. Meist bieten diese Gruben schon nach wenigen Jahren andere, bessere oder schlechtere Aufschlüsse. Mein interstdialer Würm-Verwitterungsboden ist natürlich nicht in $\mathrm{jeder}$ Grube $\mathrm{im} \mathrm{me} \mathrm{r}$ als zusammenhängende Bodenschicht durchlaufend zu sehen. Herr BüDEL spricht daher mit Recht von den Abtragungsfolgen des über meinen Boden hinweggegangenen Inlandeises. Es besteht aber die manche Kollegen vielleicht überraschende Tatsache, daß diese Abtragung des lockeren Verwitterungsbodens a uf $\mathrm{d} \in \mathrm{r}$ weiten $\mathrm{Eb}$ en e des Murnauer Würmschotters so we nig wirksam gewesen ist. Die Grundmoräne schob sich mit glatter $\mathrm{Schnittfläche} \mathrm{weithin} \mathrm{über} \mathrm{den} \mathrm{kalkfreien} \mathrm{braunen} \mathrm{Boden} \mathrm{mit} \mathrm{seinen} \mathrm{Verwitte-}$ rungstrichtern. Dagegen ist der hellgraue Geschiebemergel, also die aufliegende Grundmoräne des letzten Hauptwürmvorstoßes, sehr kalkreich, schließt mitunter Fetzen oder Fahnen meines Bodens ein, von dem manchmal nur noch die basalen Trichter übriggeblieben sind. Daraus, daß Herrn BüDEL an einer Stelle 1957 meinen Boden nicht zu finden vermochte, konnte ich ihm angesichts des raschen Gruben-Abbaues 1961, S. 45, natürlich keinen Vorwurf machen.

Die Grundfrage ist und bleibt: Gibt es in unserem vereist gewesenen Voralpenland interstadiale Würmböden oder nicht? Weil ich diese erstmals aufgefunden, mit genügender Kritik beschrieben und analysieren lassen konnte, kann ich diese Frage bejahen. Keiner der Kollegen, denen ich im Gelände den Boden zeigte, zweifelte denn auch an dem interstadialen Alter.

Wenn Herr Büdel sagt, er habe den Boden „sehr wohl im Gelände gefunden“, so ist die Sache gut. Daß dieser Fund aber nun "die von Kraus gemeinten Sedimentspuren“ im 
Gelände seien, die anders, nämlich als Folge rezenter Verwitterung zu deuten wären, dieser Meinung kann ich nicht beistimmen. Denn nach meinen langen Arbeiten im chemischen und bodenkundlichen Laboratorium der Technischen Hochschule München, im Gelände während meiner Doktorarbeit, als Kriegsgeologe, in der Zeit meines Lehrauftrages für "Geologische Bodenkunde“ an der Universität Königsberg/Pr., konnte ich während eines jeden der 40 vergangenen Sommer in den verschiedenen Gebieten Europas, von Nordafrika und in der Türkei kartieren oder genauer beobachten. Bei ausgedehnten angewandtgeologischen Arbeiten sah ich eine Unzahl frischer Bodenanschnitte: $\mathrm{Ni}$ e mals fand ich beweisende Anzeichen dafür, daß irgendwo eine von Herrn BüDEL ad hoc konstruierte Tief-Verwitterung einen dem Boden der Erdoberfläche entsprechenden Verwitterungsboden habe schaffen können. Daß nämlich z. B. unter verwitterter, sehr fester Grundmoräne von über $50 \mathrm{~cm}$ Dicke -- wie viel Geschiebemergel-Aufschlüsse konnte ich schon untersuchen! - eine klimatisch bedingte Verwitterung hinabreichen könnte und sich hier über einem durchlässigen Schotter etwa durch die Wurzeln großer Bäume horizontal ausbreitete, daß so ein entkalkter Boden in 14 Kiesgruben entstehen konnte. Auch in der Literatur kenne ich keine einwandfreien Beispiele.

Über einen solchen Versuch, mein Interstadial in einen rezenten Boden zu verwandeln, kann ich auf Grund meiner Erfahrungen keine weiteren Worte verlieren. Darum kann ich mich auch nicht mit Gedanken beschäftigen, die ausgehen von der unrichtigen Meinung, es gäbe in der Murnauer Gegend keinen interstadialen Boden - zumal jetzt auch von anderer Seite schon mehrfach dieser Boden in dem ehemals vereisten Voralpenland im $\mathrm{W}$ und $\mathrm{O}$ aufgefunden wurde.

Was diesbezüglich Herrn BüDELs ausführlichere Untersuchung von 1960 betrifft, so behandelt sie regional sehr viel ausgedehntere und allgemeinere Klimafragen. Mir aber ging es darum:

1. Die feste, von mir bearbeitete Beobachtungsgrundlage vor allem des jüngeren Würm-Interstadials zur Geltung zu bringen;

2. die Beziehungen zwischen den zwei interstadialen Würm-Landoberflächen (E. EBERs 1960) innerhalb des nordalpinen Vorlandvereisungsgebietes zu jenen im östlichen Lößgebiet (Göttweig und Paudorf) zu erkunden, wobei ich die scheinbar exakte Klimakurve von Milankovitsch auch jetz.t noch nicht genügend begründet sehe;

3. die für mich zunächst überraschende zeitliche Übereinstimmung unserer würmeiszeitlichen Klimakurve mit jener in Canada (Dreimanis) und im Felsengebirge der USA (RICHMOND) aufzuzeigen.

Und nun noch von der Schmutz-Tapete, deren Entstehung durch Aufweichen der Grundmoräne mit ihrem vielen Pelit und deren hangendem Verwitterungslehm nach langem Regen, durch anschließendes Herabgleiten über meinen Boden und mehr oder weniger über die gleichfalls steile Wandfläche des Murnauer Schotters ich geschildert habe.

Diese Erscheinung konnte ich in einigen Kiesgruben z. B. am 19. 10. 1960 Frau Dr. Ebers und Herrn Dr. Richmond (Denver) zeigen. In der besonders leicht zugänglichen Kiesgrube $2 \mathrm{~km}$ O Froschhausen, die Herr BüDEL nicht besuchte, hatte jene Schmutztapete meinen Boden völlig verhüllt. Aber ein Hammerschlag auf die ausgetrocknete Tarnschicht genügte, um meinen Boden unmittelbar unter der scharfen Basalfläche der Grundmoräne freizumachen. Herr Richmond hatte in kurzem die $12 \mathrm{~m}$ lange Bodenfortsetzung horizontal aufgeschlossen, die ich als Abb. 3 1961, S. 46, publizierte.

Am gleichen Tag war auch in der Kiesgrube NO beim Dorf Riegsee die Schmutztapete über meinem Boden erst wegzuhacken. Von hier veröffentlichte Herr BüDEL als Abb. 4 (Sept. 1956) und als Abb. 5 (April 1957) dieselbe Grubenwand-Ansicht. Diese Grube 
wurde seit 1953 durch Dr. Lebküchner, Dr. Haber, Dr. Buchardt und durch mich oft besucht. Nie erwies sich die wirklich rezente Verwitterungsrinde dicker als $25 \mathrm{~cm}$, mit Trichtern maximal $40 \mathrm{~cm}$. So habe ich sie in Abb. 12 photographiert und S. 87, 88 vom dortigen Interstadialboden die Analyse mitgeteilt. So hat auch Herr Büdel 1957 nur eine d ü $\mathrm{n}$ e rezente Bodendecke gezeichnet (Abb. 4, 5). Scharf davon unterschieden aber zeichnete er hier außerdem nach unten bis über $1 \mathrm{~m}$ reichend noch „rezente Braunerde-Verwitterungstaschen". Das sind die uns bestbekannten Schmutzkrusten-Verbreitungen an der steilen Grubenwand. Solche bilden sich, abhängig oft von flachen Wandreliefformen, an g]eicher Stelle jedes Jahr, aber auch nicht alle, wie der Vergleich von Herrn BüDELs beiden Abbildungen erkennen läßt. Gerade in dieser Grube ist die Wand meist so hoch und steil, daß man große Mühe hat, die Schmutzschicht zu entfernen oder überhaupt genau zu untersuchen. Solche Schwierigkeiten mögen Herrn BüDEL in seiner Deutung bestärkt haben.

Es kommt aber natürlich vor, daß bei sehr dünnem Grundmoränenbelag, der dann nachwürmisch ganz verwittert ist, dieser „rezente “ Boden in den interstadialen übergeht sofern dieser an dieser Stelle erhalten war. Mit Herrn Dr. F. Weidenbach habe ich dies z. B. auch am 26. 6. 62 in Etting festgestellt.

Weshalb man immer nur mit höchstens 2 oder $3 \mathrm{~m}$ Grundmoränenbelag den jüngeren Interstadialboden sieht, ist unschwer verstehbar. Denn die Grundmoräne ist Abraum bei der Kiesgewinnung. Diese lohnt sich unter mächtigerem Abraum nicht mehr. Folglich ist derzeit aus Kiesgruben der Nachweis nicht zu liefern, daß auch unter mächtiger Grundmoräne ein würminterstadialer Boden liegt, dessen Entstehung durch eine „rezente Grundverwitterung " noch unwahrscheinlicher wäre.

Herr BüDEL erlaubte sich mir gegenüber den massiven Vorwurf einer „bewußten Erfindung “. Ich würde ihn schärfstens zurückweisen, würde ich nicht sehen, daß sich der Genannte auch diesbezüglich getäuscht hat. Denn aus dem Zusammenhang meines Textes 1961, S. 46/47, geht wohl klar hervor, daß ich in dem neuen Absatz, beginnend mit Textzeile 3 von oben, nicht mehr allein sprach von dem Bodenvorkom-

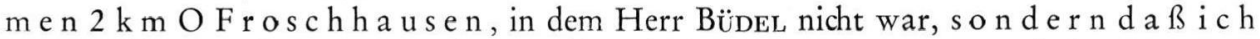
nun allgemein versuchte, die Schmutztapete, welche wegen des Pelitgehaltes der Grundmoräne in allen Kiesgruben auftritt, $\mathrm{z} u$ ke nn ze i chne n. „Deren sonderbare Gestalten zeichnete offenbar J. BüdeL in seiner Arbeit, nicht aber meinen von ihm gesuchten Boden" (bei mir 1960) kann sich selbstverständlich nur beziehen auf Zeichnungen, die Herr BüDEL wirklich publizierte, und das sind jene drei Abb. 3 bis 5 von 1957. Ich bedauere, daß der Genannte diese Schmutzschichten verwechselte mit einem rezenten Verwitterungsboden, und zwar in anderen Gruben als die von mir dargestellte Schmutztapete 1961, Abb. 3. Noch mehr aber bedauere ich, daß er mich einer bewußten Erfindung für fähig gehalten hat. Wie ich ihn kenne, darf ich hoffen, daß er diesbezüglich seinen Irrtum einsieht. Bedauerlich ist weiterhin, daß er mit seinen Worten: „Die Krönung eines solchen Verhaltens bietet schließlich Kraus' lapidare Folgerung aus dem dreifach falschen Bild, das er dem Leser von mir entrollte" mir vorwirft, ich hätte ihn selbst persönlich angegriffen. Was ich a uf seine Angriffe tat, das war die Wiedergabe der Beobachtungen, von denen sich jeder Kollege im Gelände überzeugen kann.

Bezüglich der D e u t u n g von Beobachtungen räume ich Herrn BüDEL selbstverständlich dasselbe Recht ein, das auch ich selbst beanspruche. Ich will ihn auch nicht veranlassen, meine Auffassung anzunehmen, obwohl ich ihm das empfehlen würde. Ich habe mich mitunter auch getäuscht, wollte dann aber immer der erste sein, der das zugibt. Maßgebend sind für mich die Beobachtungen. Diese zeigen, daß ich mich diesmal jedenfalls nicht getäuscht habe.

Gefreut hätte es mich, wenn Herr BüDEL, den ich wegen seiner Arbeiten durchaus schätze, angesichts solcher Deutungs-Gegensätze mit mir zusammen die Aufschlüsse unter- 
sucht hätte. Das ist die Art, Zwist und unnötige Schreibereien entbehrlich zu machen. Im übrigen wäre ich unseren gemeinsamen Fachkollegen dankbar, wenn sie sich im Zweifelsfall selbst ein Bild machen würden durch Betrachtung jener Kiesgruben und wenn sie dann helfen würden, diese Angelegenheit zu ordnen. ${ }^{1}$ )

\section{Lit eratur}

1955 E. C. Kraus: Zur Zweigliederung der südbayerischen Würmeiszeit durch eine InnerwürmVerwitterungsperiode. - Eiszeitalter und Gegenwart 6, 75-95. Öhringen/Württ. 1955.

1957 J. BüDEL: Die angebliche Zweiteilung der Würmeiszeit im Loisach-Vorland bei Murnau (Südbayern). - Stuttgarter geograph. Studien 69, Stuttgart 1957.

1960 - Die Gliederung der Würmkaltzeit. - Mitt. geograph. Gesellschaft Würzburg, Heft 8. 1960 E. Ebers: Drumlinkerne, ältere Würmschotter und das Würm-Interstadial-Profil von Hörmating/Obb. - Eiszeitalter und Gegenwart 11, 64-76. 1960.

1961 E. C. Kraus: Die beiden interstadialen Würmböden in Südbayern. - Ebenda 12, 43-54. 1961.

1962 J. BüDEL: Die beiden interstadialen Würmböden. Eine Richtigstellung. Hektographiert an beschränkten Leserkreis.

Manuskr. eingeg. 17. 6. 1962.

Anschrift des Verf.: Prof. Dr. Ernst Kraus, München-Obermenzing, Verdistraße 40.

1) Herr Professor Dr. P. Woldstedt gab mir inzwischen freundlichst Kenntnis von der Korrektur des ihm von Herrn Professor Dr. J. BüDEL zum Druck vorgelegten Aufsatzes. Im Vorstehenden glaube ich zugleich die wesentlichsten Punkte dieses Aufsatzes beantwortet zu haben. 